\title{
Anesthetic Management for Spine Surgery in a Patient with Left Internal Carotid Artery Occlusion and Penetrating Aortic Ulcer: A Case Report
}

\author{
Misoon Lee, Dasom Choi, Bon Sung Koo, Sung-Hwan Cho \\ Department of Anesthesiology and Pain Medicine, Soonchunhyang University Bucheon Hospital, Bucheon, Korea
}

\begin{abstract}
A key challenge of anesthesia is to provide patients with safe and optimized anesthetic management to improve prognosis and minimize mortality and morbidity. To this end, the anesthesiologist should comprehensively understand the patient's physical status through pre-anesthetic assessment and carefully monitor the patient during surgery. Several types of novel patient-monitoring devices may be useful to achieve this purpose. We report a case of anesthetic management in a patient with left internal carotid artery occlusion and penetrating aortic ulcer.
\end{abstract}

Keywords: Carotid stenosis; Brain ischemia; Physiologic monitoring; Oximetry; Aorta; Cerebrovascular circulation; Case report

\section{INTRODUCTION}

Although the incidence of ischemic stroke in asymptomatic patients with carotid stenosis is low, the incidence of perioperative stroke in patients with carotid stenosis is 36 times that of healthy individuals [1]. Therefore, it is important to maintain cerebral blood flow and oxygenation during anesthesia in patients with carotid artery stenosis, and events that affect cerebral blood flow, such as hypoxia and hypotension, should be prevented. Monitoring of cerebral oxygen saturation through regional cerebral oxygen saturation $\left(\mathrm{rSO}_{2}\right)$ measurement is useful for detecting changes in cerebral blood flow during surgery. However, for anesthesia of patients with various underlying diseases, it is necessary not only to assess each disease individually but also to evaluate comprehensive physical status, and optimal management should be provided. Here, we report a case of anesthetic management in a patient with left internal carotid artery occlusion and penetrating aortic ulcer (PAU).

\section{CASE REPORT}

A 76-year-old female $(152 \mathrm{~cm}, 63 \mathrm{~kg}$, American Society of Anes- thesiologists status III) was admitted for elective posterior decompression of L1-3, corpectomy of L2, and posterolateral fixation of T12-L3 for a compression fracture of L2. Her past medical history included high blood pressure and total occlusion of the left internal carotid artery, which was being treated with angiotensin receptor blockers, calcium channel blockers, diuretics, and aspirin. Preoperative laboratory tests showed no abnormal results, but left ventricular (LV) hypertrophy was observed in electrocardiography. Preoperative transthoracic echocardiography revealed concentric LV remodeling and relaxation abnormality of the LV filling pattern $\left(E^{\prime} / E^{\prime}=18.4\right)$, with an ejection fraction of $56 \%$.

Magnetic resonance imaging of the brain and femoral carotid angiography were performed to assess the patient's perioperative risk. Total left artery occlusion and collateral blood flow were observed (Fig. 1). Furthermore, during femoral carotid angiography, residual contrast agent was detected around the innominate artery, suggesting the possibility of aortic dissection. Thus, aortic dissection computed tomography (CT) was additionally performed for differential diagnosis. A PAU with approximately $14 \mathrm{~mm}$ of length and $8 \mathrm{~mm}$ of depth of the distal aortic arch and severe calcification with plaque of the innominate artery were revealed (Fig. 2). The cardiovascular surgeon who reviewed these abnormal
Correspondence to: Sung-Hwan Cho

Department of Anesthesiology and Pain Medicine, Soonchunhyang University Bucheon Hospital, 170 Jomaru-ro, Wonmi-gu, Bucheon 14584, Korea

Tel: +82-32-621-5339, Fax: +82-32-621-5322, E-mail: singaring@schmc.ac.kr

Received: Nov. 3, 2021 / Accepted after revision: Nov. 10, 2021
(C) 2021 Soonchunhyang Medical Research Institute This is an Open Access article distributed under the terms of the Creative Commons Attribution Non-Commercial License (https://creativecommons.org/licenses/by-nc/4.0/). 

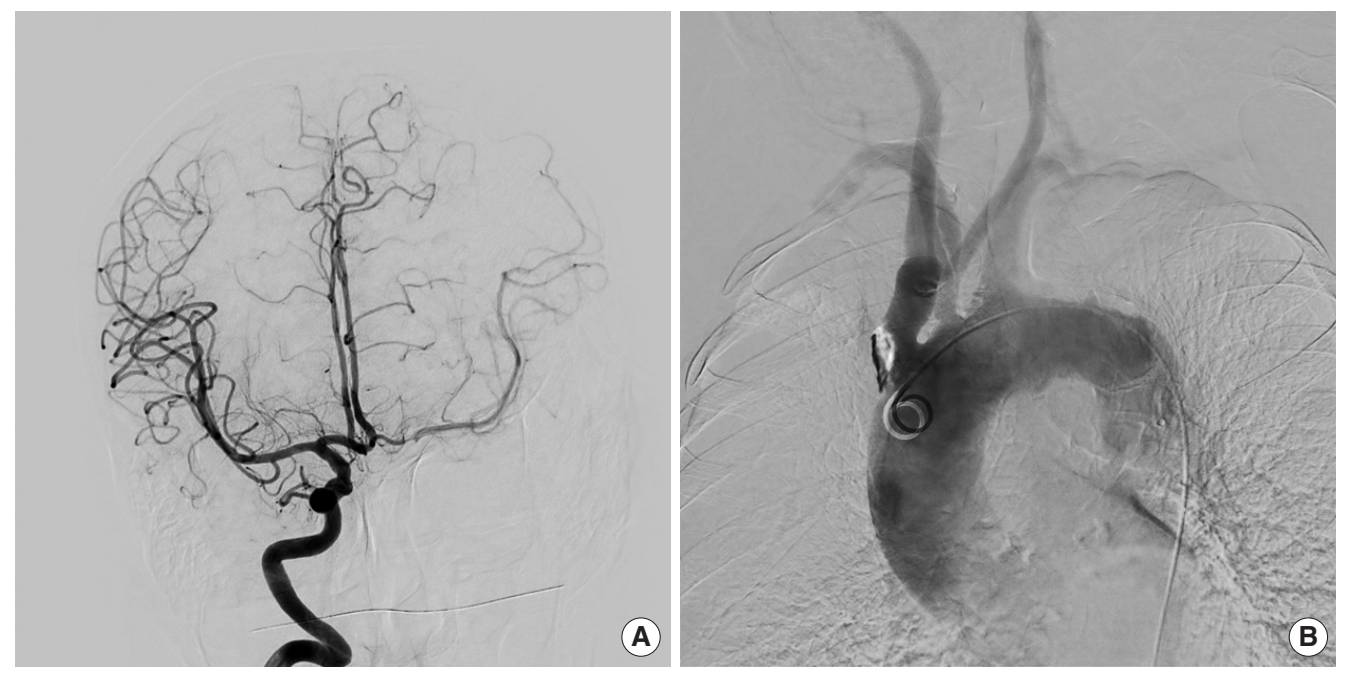

Fig. 1. Angiography of carotid artery findings. (A) Total occlusion of left internal carotid artery with collateral flow. (B) Residual contrast agent around the innominate artery was seen.
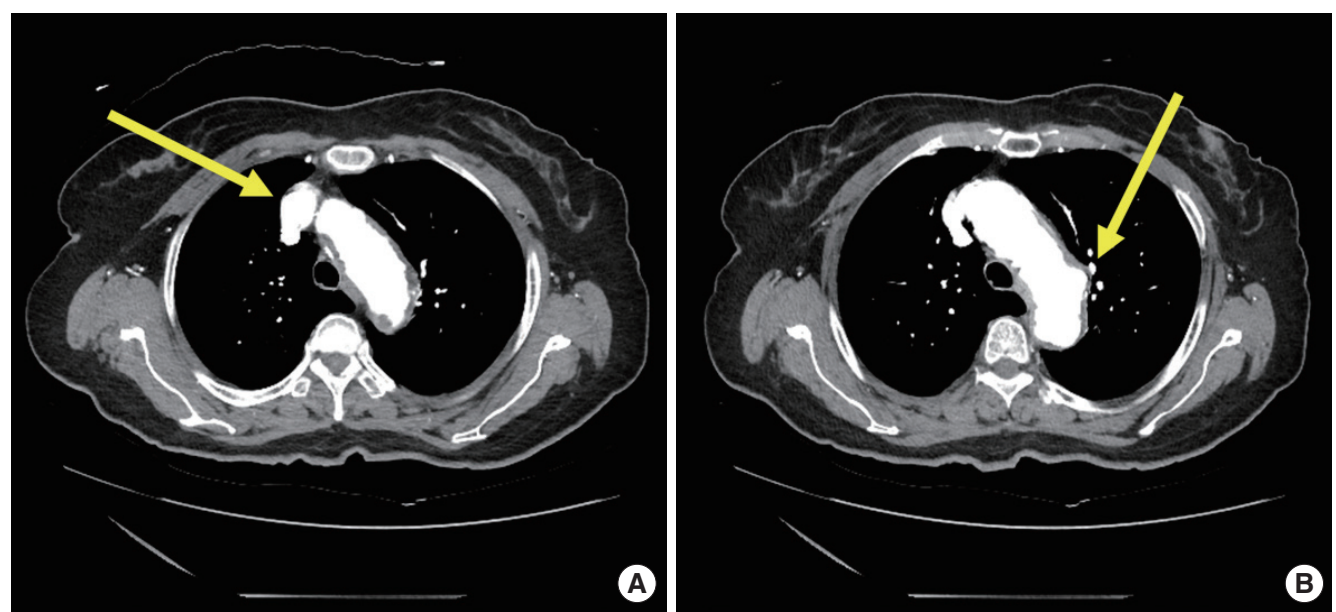

Fig. 2. Aortic dissection computed tomography findings. (A) Severe calcification with plaques of innominate artery (yellow arrow). (B) Penetrating aortic ulcer in distal aortic arch (yellow arrow).

findings recommended maintaining the perioperative systolic blood pressure below $130 \mathrm{~mm} \mathrm{Hg}$.

Upon arrival at the operating room, standard monitors, including a noninvasive blood pressure monitor, three-lead electrocardiography, pulse oximetry, and a bispectral index (BIS) monitor, were attached to the patient. Then, a cerebral oximeter $\left(\mathrm{rSO}_{2}, \mathrm{INVOS}\right.$ Cerebral/Somatic Oximeter; Covidien-Medtronic, Boulder, CO, USA) was attached for continuous monitoring of $\mathrm{rSO}_{2}$. General anesthesia was induced with intravenous lidocaine (40 mg), 2\% propofol, and remifentanil. Propofol and remifentanil were administered via infusion pump (Agilia; Fresenius Kabi, Bad Homburg, Germany), using the Marsh pharmacokinetic model and
Minto model, respectively.

Esmolol (20 mg) was intravenously administered to prevent sudden change of blood pressure and heart rate before endotracheal intubation. After anesthetic induction, a 22G catheter was inserted into the left radial artery, and then the FloTrac/Vigileo system (Edwards Lifesciences, Irvine, CA, USA) was used for continuous monitoring of arterial blood pressure, cardiac output, and stroke volume. A central venous catheter was subsequently inserted into the right internal jugular vein using ultrasound guidance. Anesthesia was maintained with continuous intravenous administration of propofol, remifentanil, and rocuronium. During anesthesia, we adjusted the target concentrations of propofol and remi- 


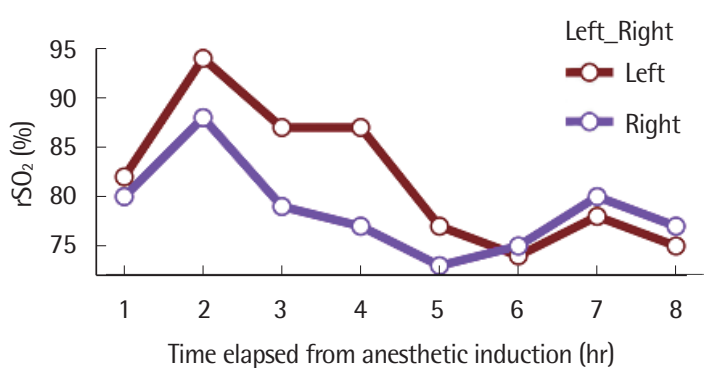

Fig. 3. Values of intraoperative regional cerebral oxygen saturation $\left(\mathrm{rSO}_{2}\right)$ measurement.

fentanil to maintain the BIS at 40-60 and set up the mechanical ventilator according to the arterial blood gas analysis findings and end-tidal carbon dioxide levels to maintain mild hypocapnia to prevent the steal phenomenon. In addition, according to the recommendation of the cardiovascular surgeon, esmolol, nicardipine, ephedrine, and phenylephrine were administered intravenously when a decrease or increase in blood pressure was necessary to maintain the target systolic blood pressure below $130 \mathrm{~mm} \mathrm{Hg}$. After the operation was completed, the patients' blood pressure gradually increased as the propofol and remifentanil were stopped. Therefore, intravenous esmolol and nicardipine were administered under careful monitoring of the hemodynamic changes during the anesthetic emergence.

Total anesthesia time was 9 hours and 41 minutes. During the operation, a total of 6 units of packed red blood cells and 6 units of fresh frozen plasma were transfused in the operating room. All hemodynamic parameters were stable, and $\mathrm{rSO}_{2}$ was maintained above $70 \%$ (Fig. 3). After the end of surgery, the patient was transferred to the postoperative care unit, where she stayed for $45 \mathrm{~min}$ utes, and then was moved to the ward without any immediate postoperative complications.

This study was approved by the institutional review board of Soonchunhyang University Bucheon Hospital (approval no., SCHBC 2021-11-004). The board waived the requirement for patient' informed consent because this study was a retrospective medical record review.

\section{DISCUSSION}

Carotid artery stenosis significantly increases the risk of stroke [1]. The perioperative stroke risk in patients with more than $50 \%$ carotid artery stenosis is $3.6 \%$, compared to $0.1 \%$ in healthy individuals [2]. According to the literature, the degree of stenosis is most directly related to the factor that increases this stroke risk in asymptomatic carotid stenosis [3]. For patients with carotid artery stenosis, it is important to optimize brain perfusion and minimize myocardial stress [4]. Because intraoperative hypotension can worsen neurological outcomes, proper blood pressure management is required [5]. Several factors are known to decrease cerebral oxygenation during anesthesia. Decreases in cardiac output, changes in blood acid-base status, bleeding, and arterial inflow/venous outflow obstruction induced decreases in cerebral blood flow. In addition, changes in hemoglobin concentration and saturation, pulmonary function, and inhaled oxygen concentrations affect the decrease in oxygen content, which may lead to decreases in cerebral blood flow [6]. These factors can occur at any time during general anesthesia, so the patient should be carefully monitored. For this, the application of monitoring devices that can quantitatively measure $\mathrm{rSO}_{2}$, such as near-infrared spectroscopy (NIRS), may be helpful. [7]. However, due to its low sensitivity and specificity, NIRS should not be used alone in the diagnosis of cerebral ischemia [8].

Our patient had carotid artery stenosis accompanied by PAU discovered incidentally during preoperative workup. Aortic dissection, intramural hematoma, and PAU are considered part of the same spectrum of acute aortic syndrome [9]. PAU is defined as a condition in which ulceration of atherosclerotic plaques in the aorta wall penetrates the media through the intima [10]. There may be no symptoms in the early stages, but as the disease progresses, it can cause intramural hematoma and even life-threatening conditions such as aortic dissection or rupture. The treatment of PAU is determined according to the presence or absence of symptoms and the location of the lesion. For lesions of the descending aorta, if asymptomatic, aggressive antihypertensive medical therapy and regular follow-up can be applied instead of surgical treatment [11]. In our case, conservative treatment and annual CT follow-up were chosen because of the asymptomatic, small lesions in the descending aortic arch.

Therefore, two important considerations in the anesthetic management of this patient were to prevent brain ischemic injury and to prevent acute exacerbation of PAU in the perioperative period. Although the patient had total occlusion of the left internal carotid artery, angiography revealed that sufficient cerebral blood flow was supplied by collateral circulation. To prevent damage to the carotid artery during central venous catheter insertion, the procedure was performed with visualization of the internal jugular vein and ca- 
rotid artery using ultrasound. Then, continuous $\mathrm{rSO}_{2}$ monitoring was used for the early detection of desaturation. Because the surgery was quite long, there were risks of anemia and hypovolemia following massive hemorrhage and acid-base balance changes. Therefore, cardiac output and volume status were continuously monitored using the FloTrac/Vigileo system. Moreover, we conducted arterial blood gas analysis at regular intervals to obtain arterial oxygen tension, arterial carbon dioxide tension, oxygen saturation, and hemoglobin concentration. To prevent sudden hemodynamic changes due to surgical stimulation, we maintained an appropriate depth of anesthesia via BIS measurement.

In conclusion, for the safe anesthetic management of patients at high risk for complications, it is necessary to predict risk factors in advance through a pre-anesthesia evaluation. During anesthesia, careful patient monitoring is essential, and when complications occur, anesthesiologists need to be prepared to recognize them and respond immediately. In addition, use of appropriate patientmonitoring devices may be helpful in anesthetic management of high-risk patients.

\section{CONFLICT OF INTEREST}

No potential conflict of interest relevant to this article was reported.

\section{REFERENCES}

1. Nadareishvili ZG, Rothwell PM, Beletsky V, Pagniello A, Norris JW. Longterm risk of stroke and other vascular events in patients with asymptomatic carotid artery stenosis. Arch Neurol 2002;59:1162-6.

2. Evans BA, Wijdicks EF. High-grade carotid stenosis detected before general surgery: is endarterectomy indicated? Neurology 2001;57:1328-30.

3. Inzitari D, Eliasziw M, Gates P, Sharpe BL, Chan RK, Meldrum HE, et al. The causes and risk of stroke in patients with asymptomatic internal-carotid-artery stenosis. North American Symptomatic Carotid Endarterectomy Trial Collaborators. N Engl J Med 2000;342:1693-700.

4. Erickson KM, Cole DJ. Carotid artery disease: stenting vs endarterectomy. Br J Anaesth 2010;105 Suppl 1:i34-49.

5. Yu Q, Qi J, Wang Y. Intraoperative hypotension and neurological outcomes. Curr Opin Anaesthesiol 2020;33:646-50.

6. Tosh W, Patteril M. Cerebral oximetry. Bja Educ 2016;16:417-21.

7. Ali J, Cody J, Maldonado Y, Ramakrishna H. Near-infrared spectroscopy (NIRS) for cerebral and tissue oximetry: analysis of evolving applications. J Cardiothorac Vasc Anesth 2021 Jul 10 [Epub]. https://doi.org/10.1053/ j.jvca.2021.07.015.

8. Rigamonti A, Scandroglio M, Minicucci F, Magrin S, Carozzo A, Casati A. A clinical evaluation of near-infrared cerebral oximetry in the awake patient to monitor cerebral perfusion during carotid endarterectomy. J Clin Anesth 2005;17:426-30.

9. Tsai TT, Nienaber CA, Eagle KA. Acute aortic syndromes. Circulation 2005;112:3802-13.

10. Stanson AW, Kazmier FJ, Hollier LH, Edwards WD, Pairolero PC, Sheedy PF, et al. Penetrating atherosclerotic ulcers of the thoracic aorta: natural history and clinicopathologic correlations. Ann Vasc Surg 1986;1:15-23.

11. Henn MC, Lawrance CP, Braverman AC, Sanchez L, Lawton JS. Medical and surgical management of a descending aorta penetrating atherosclerotic ulcer and associated ascending intramural hematoma. Aorta (Stamford) 2014;2:77-81 . 